\title{
Candidemia and its Risk Factors in Neonates and Children
}

\author{
Azam Fattahi ${ }^{1}$, Ensieh Lotfali (ib ${ }^{2}$, Hossein Masoumi-Asl ${ }^{3,}$, , Shirin Sayyahfar ${ }^{3}$, Majid Kalani $^{3}$, \\ Mohammad Rafi Khourgami (ib ${ }^{4}$ and Zeynab Yassin ${ }^{5}$ \\ ${ }^{1}$ Center for Research and Training in Skin Diseases and Leprosy, Tehran University of Medical Sciences, Tehran, Iran \\ ${ }^{2}$ Department of Medical Parasitology and Mycology, School of Medicine, Shahid Beheshti University of Medical Sciences, Tehran, Iran \\ ${ }^{3}$ Research Center of Pediatric Infectious Diseases, Institute of Immunology and Infectious Diseases, Iran University of Medical Sciences, Tehran, Iran \\ ${ }^{4}$ Rajaie Cardiovascular, Medical, and Research Center, Rajaie Hospital, Iran University of Medical Sciences, Tehran, Iran \\ ${ }^{5}$ Antimicrobial Resistance Research Center, Iran University of Medical Sciences, Tehran, Iran \\ "Corresponding author: Research Center of Pediatric Infectious Diseases, Institute of Immunology and Infectious Diseases, Iran University of Medical Sciences, Tehran, Iran. \\ Email: dr_masoumiasl@yahoo.com
}

Received 2020 February 05; Revised 2020 June 08; Accepted 2020 June 28.

\begin{abstract}
Objectives: The present study was conducted to raise attention to the frequency of Candida spp. and evaluation of risk factors of candidemia in hospitalized neonates and children.

Methods: Identification of Candida at species level was done using the PCR-RFLP method. The Candida albicans complex and Candida parapsilosis complex were differentiated using the HWP1 gene amplification and PCR-RFLP with NlaIII restriction enzyme, respectively.

Results: Out of 75 blood culture specimens, 42 (84\%) cases were positive for Candida spp. of whom 30 (71.42\%) and 12 (28.57\%) cases were female and male, respectively. Thirty-two (76\%) candidemia were presented in pediatrics with 6 years up to 12 years, 10 ( $23.80 \%)$ in neonates of one month or less. In the present study, Candida parapsilosis $(\mathrm{n}=25 ; 59.52 \%)$ was the most prevalent isolated species followed by C. albicans ( $n=11 ; 26.19 \%)$, C. tropicalis $(n=4 ; 9.52 \%)$, and Candida glabrata $(n=2 ; 4.76 \%)$.

Conclusions: According to potentially dangerous complications of bloodstream infection by Candida spp. in neonates and children, it is necessary to identify and eliminate the underlying conditions and risk factors of this disease.
\end{abstract}

Keywords: Candida Species, Candidemia, Risk Factor, Neonates, Children

\section{Background}

Candidemia in neonates and children can lead to severe morbidity, and it can be even fatal despite appropriate treatment (1). According to the literature, the epidemiologic pattern and frequency of pediatric candidal infection differs from adults (2); thereby needing comprehensive strategies to the management of candidemia (2). Candida spp. is known as the third to fourth most common pathogen cause of nosocomial bloodstream infections (BSI) $(3,4)$. This destructive infection is mainly caused by, Candida albicans $(5,6)$. Currently, the epidemiology of the candidemia is changing, and we are facing the emergence of non-C. albicans (NAC) spp., including Candida parapsilosis, Candida tropicalis, Candida glabrata, and Candida krusei (7). Candida parapsilosis is considered the predominant causative agent of candidemia in pediatric oncologic disorders (2). Differences in Candida spp. are related to disease severity and diversity in antifungal susceptibility, patterns; therefore, understanding the type of species is clinically important.

Only limited information about burden of infection and risk factors are available in neonates and pediatrics in Iran and further study is required to address its burden (5, $6,8-10)$.

\section{Objectives}

The present study was conducted to raise attention to the frequency of Candida spp. and evaluation of risk factors in hospitalized neonates and children that were suspected of having invasive candidiasis (IC).

\section{Methods}

\subsection{Study Areas and Subjects}

This cross-sectional study was conducted from 2017 to 2018. The blood samples were obtained from each patient using a sterile syringe and directly infused into biphasic 
blood media. Seventy-five blood culture samples were collected from neonates and children $\leq 14$ years old who were hospitalized and suspected of fungal infection. Bacterial positive samples were excluded from the study.

\subsection{Conventional Identification}

Blood samples were inoculated into aerobic culture medium bottles (BACTEC Peds Plus/F Culture Vials, Ireland) followed by incubation for a period of 5 days in the automated blood culture system BACTEC 9120 (Becton Dickinson, Spark, MD, USA). The candidemia was defined as having more than one positive culture specimen. Each episode of IC was considered a new case if at least one month would have been passed from a previously treated occurrence (3). The positive cultures were subcultured on blood agar (Merck, Darmstadt, Germany) at $35^{\circ} \mathrm{C}$ and checked each day for up to five days. To access the pure colony, the positive cultures were subcultured on CHROMagar (CHROMagar Paris, France) at $35^{\circ} \mathrm{C}$ for 24 hours.

\subsection{Molecular Identification}

All isolates were cultured on yeast extract peptone dextrose (YEPD) agar (Merck, Germany) at $37^{\circ} \mathrm{C}$ for 24 hours. DNA was extracted using the genomic DNA extract kit (GeneAll, Korea).

Definite identification of Candida spp. was confirmed by the PCR-restriction fragment length polymorphism (RFLP) approach based on the amplification of ITS1-5.8 SrDNA-ITS2 region with pun fungal primers ITS1-ITS4 that were synthesized by Bioneer Company (Korea) and enzymatic digestion. The PCR was conducted with a mixture included $1 \mu \mathrm{L}$ of extracted DNA, $10 \mu \mathrm{L}$ of Taq DNA Polymerase Master Mix RED (Ampliqon, Denmark), $1 \mu \mathrm{L}$ of each ITS1 (5'-TCCGTAGGTGAACCTGCGG-3') and ITS4 (5'TCCTCCGCTTATTGATATGC -3') pair primers (11), $\mathrm{H}_{2} \mathrm{O}$ up to $25 \mu \mathrm{L}$. The PCR program as follow as; $95^{\circ} \mathrm{C}$ for 5 minutes, 35 cycles of denaturation for 5 minutes at $94^{\circ} \mathrm{C}$, annealing for 1 minute at $58^{\circ} \mathrm{C}$, an extension for 1 minute at $72^{\circ} \mathrm{C}$, with a final extension of 7 minutes at $72^{\circ} \mathrm{C}$. The amplicons were visualized using 1.5\% agarose gel electrophoresis in TBE buffer (Merck, Germany) and stained with safe stain (Waltham, USA). The products were subjected to the MSP1 restriction enzyme(Fermentas, USA). Restriction fragments were separated by $2 \%$ agarose gel electrophoresis for 1 hour. The C. albicans complex and C. parapsilosis complex were differentiated using the HWP1 gene amplification and PCR-RFLP with NlaIII restriction enzyme, respectively $(12,13)$. The PCR program was similar to ITS1-5.8 SrDNA- ITS2 PCR with a minor difference in annealing temperature.

\section{Results}

Out of 75 blood cultures, 42 (84\%) cases were positive for Candida spp. growth of whom 30 (71.42\%) were female, and $12(28.57 \%)$ were male. Thirty-two (76\%) candidemia were presented in pediatrics with 6 years up to 12 years, $10(23.80 \%)$ in neonates of one month or less. The patients had received several wide-spectrum antibiotics. There was evidence of empiric antifungal treatment based on medical records, including caspofungin, voriconazole, amphotericin B and it was not qualified for antifungal therapy. Nineteen $(45.23 \%)$ of pediatrics had hematological malignancy and 2 (7.1\%) had heart disease and remains had other non-leukemia related disease or neonate. The evidence of neutropenia, low weight $<1500$, long-stay hospitalization, chemotherapy, history of surgery, central venous catheters (CVC) use were also seen.

The amplification pattern of the ITS1-5.8 SrDNA-ITS2 region of Candida spp. using the ITS1-ITS4 primers is shown in Figure 1. Subsequently, all isolates were subjected to the digestion by MSP enzyme to precise identification (Figure 2). All of the $C$. albicans complex strains were identified as C. albicans with HWP1 gene primers (Figure 3).

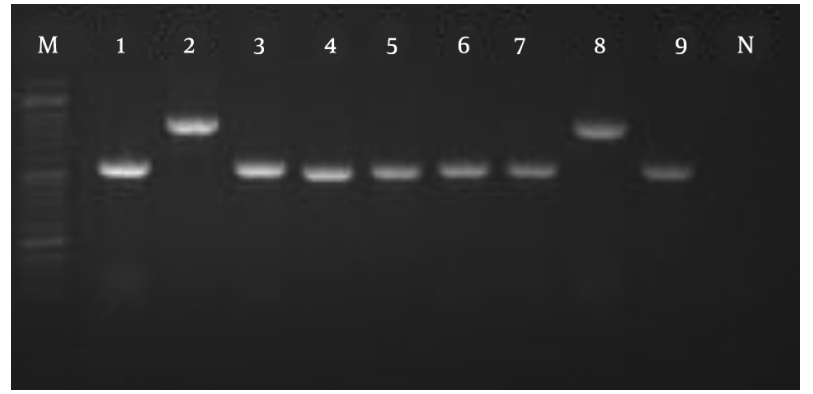

Figure 1. The amplification pattern of ITS1-5.8 SrDNA-ITS2 region of Candida spp. using the pun fungal primers. M: 100 bp DNA size marker.

In the present study, C. parapsilosis ( $\mathrm{n}=25 ; 59.52 \%$ ) was known as the most prevalent isolated species followed by C. albicans ( $\mathrm{n}=11 ; 26.19 \%)$, C. tropicalis ( $\mathrm{n}=4 ; 9.52 \%)$, C. glabrata $(\mathrm{n}=2 ; 4.76 \%)$ (Figure 4$)$.

\section{Discussion}

The number of uncommon Candida spp. isolated from clinical samples is increasing, and invasive candidiasis (IC) caused by these species may paradoxically be the result of advances in medical care (6). Regarding higher resistance to fluconazole and echinocandins in non-albicans Candida 

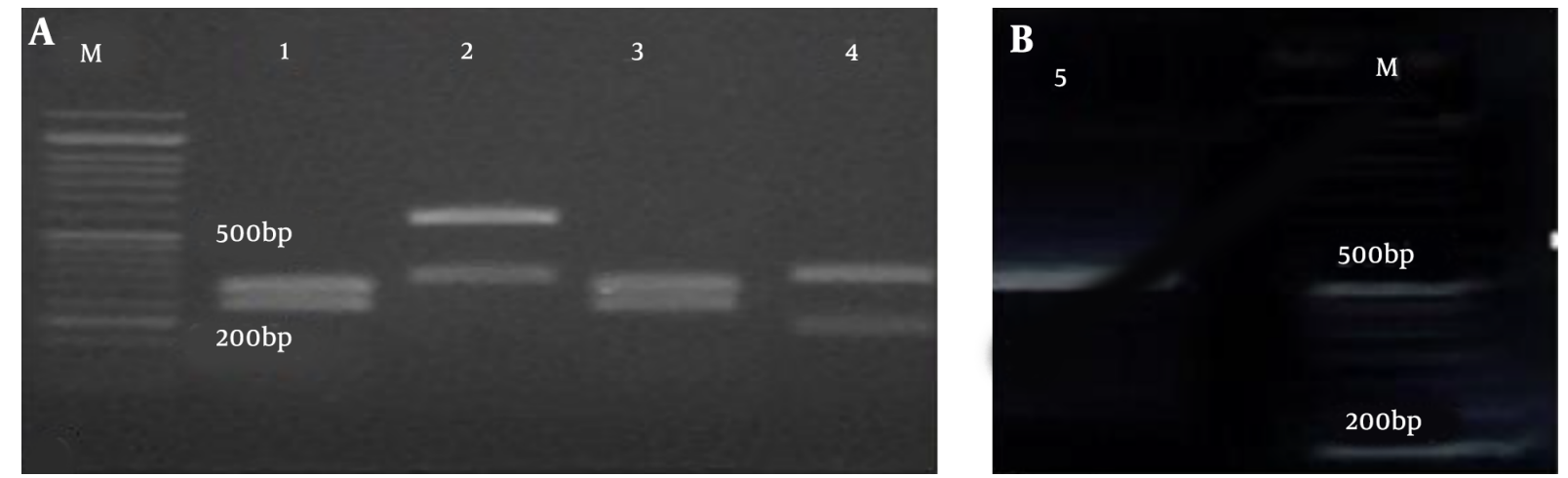

Figure 2. RFLP pattern with MspI restriction enzyme: A, C. albicans (297 - 238 bp): 1, 3; C. glabrata (314 - 557 bp): 2; C. tropicalis (184 - 340 bp): 4; B, C. parapsilosis (520 bp):5. M: 100 bp DNA size marker.

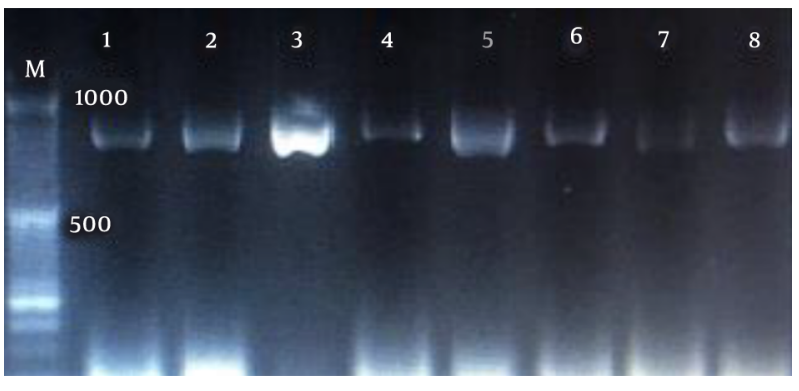

$\overline{\text { Figure 3. The amplification pattern of HWP1 genes in C. albicans complex. M:100 bp }}$ DNA size marker.

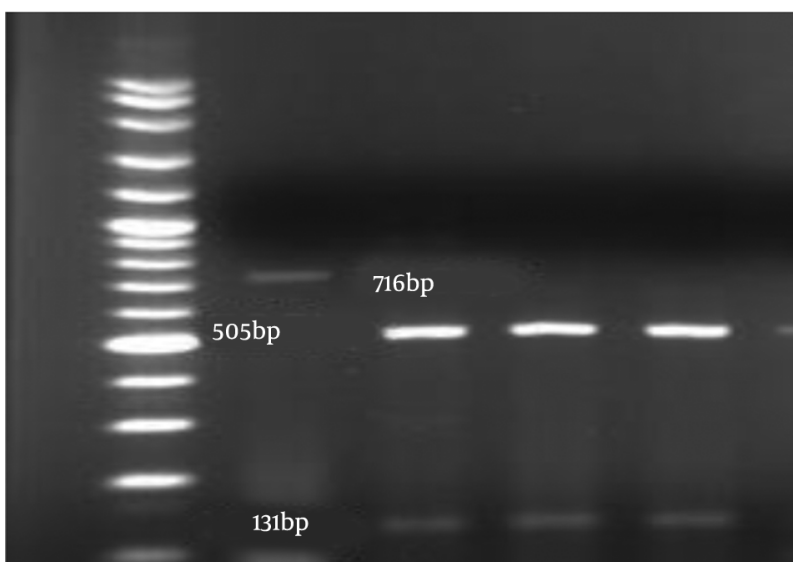

Figure 4. Agarose gel electrophoresis of RFLP-PCR products of C. parapsilosis after digestion with NlaIII. Lane M, 100 bp DNA size marker; lane 1, C. parapsilosis ATCC 22019 without effect enzyme (716 bp); lanes 2 - 8 are C. parapsilosis (131 and 505 bp).

spp. (NAC), identification of Candida spp. is necessary for appropriate management of infection.

As mentioned before, the studied population had sev- eral risk factors for developing candidemia. In the present study, C. parapsilosis ( $\mathrm{n}=25 ; 59.52 \%$ ) known as the most prevalent isolated species followed by C. albicans $(\mathrm{n}=11$; $26.19 \%)$, C. tropicalis ( $\mathrm{n}=4 ; 9.52 \%)$, and C. glabrata $(\mathrm{n}=2$; 4.76\%). These findings were consistent with previous reports that indicated C. parapsilosis complex was considered the predominant causative agent of candidemia in both neonates and pediatrics $(5,9,10,12,13)$. In some multicenter studies, $C$. albicans was considered the most frequent species in pediatrics $(6,8,10)$.

Regarding the risk factors, there was no significant difference between the two groups of candidiasis by C. albicans and NAC $(\mathrm{P}<0.01)$. A study by Juster-Reicher et al. also revealed no difference between the two groups with candidemia caused by C. albicans and C. parapsilosis $(\mathrm{P}<$ 0.01) (14) considering the risk factors such as CVC, oncologic disorders, and chemotherapy. However, there are several studies showing a higher frequency of bloodstream infections caused by C. parapsilosis in CVC usage $(5,12,15-17)$. In contrast to the pediatric population, low birth weight (LBW), and very low birth weight (VLBW) neonates were more associated with candidemia caused by C. parapsilosis $(16,18)$.

In contrast to previous studies $(6,19)$ in pediatric C. tropicalis is the third common strains were isolated in candidemia in the present study. In line with previous research, further analyses revealed that $C$. tropicalis candidemia has been associated with neutropenia and leukemia (20).

C. glabrata is rarely seen in neonates and children. However, in a study performed on immunocompromised children, C. glabrata was isolated as the second frequent cause of candidemia (19). Contrary to Charsizadeh et al. 
study (6), we did not isolate C. glabrata in blood samples in the neonatal intensive care unit (NICU). Two C. glabrata strains were isolated in patients suffering from acute lymphoblastic leukemia (ALL), the later admitted in pediatric intensive care unit (PICU) and both of them had CVC. Kara et al. (18) isolated C. glabrata from pediatrics that had infective endocarditis. In a study from Australia, neonates had a higher rate (9.1\%) than pediatrics (10). Since Candida spp., which were once considered to be harmless, can now cause candidemia and significant morbidity and mortality in patients residing in intensive care units, identifying the underlying conditions and risk factors must be noticed seriously. Candida bloodstream infection might be the result of selective pressure of some antifungal agents, previous wide spectrum antibacterial therapy, multiple intravascular catheters, parenteral nutrition, and mechanical ventilation. However, some studies found no relationship between Candida serotypes and underlying diagnosis, risk factors, clinical features, and outcomes (20-23).

\subsection{Conclusions}

According to potentially dangerous complications of bloodstream infection by Candida spp. in neonates and children, it is necessary to identify and eliminate the underlying conditions and risk factors of this disease within an appropriate framework.

\section{Footnotes}

Authors' Contribution: Study concept and design and technical supervision: AF and HMA. Obtaining the samples from patients and interpretation: SS, MK, MRK, and Z.Y. Technical support: AF. Acquisition of data and drafting the manuscript: EL. and SS. Manuscript writing: AF. Critical revision of the manuscript: EL.

Conflict of Interests: The authors declare that there is no conflict of interest.

Funding/Support: This research has been financially supported by Iran University of Medical Sciences, Tehran, Iran (grant no.: 26590).

\section{References}

1. Steinbach WJ. Epidemiology of invasive fungal infections in neonates and children. Clin Microbiol Infect. 2010;16(9):1321-7. doi: 10.1111/j.14690691.2010.03288.x. [PubMed: 20840541].

2. Roilides E, Walsh TJ. Invasive fungal infections in paediatric patients. Clin Microbiol Infect. 2010;16(9):1319-20. doi: 10.1111/j.14690691.2010.03337.x. [PubMed: 20678174].

3. Wisplinghoff H, Bischoff T, Tallent SM, Seifert H, Wenzel RP, Edmond MB. Nosocomial bloodstream infections in US hospitals: analysis of 24,179 cases from a prospective nationwide surveillance study. Clin Infect Dis. 2004;39(3):309-17. doi: 10.1086/421946. [PubMed: 15306996].
4. Steinbach WJ, Roilides E, Berman D, Hoffman JA, Groll AH, Bin-Hussain I, et al. Results from a prospective, international, epidemiologic study of invasive candidiasis in children and neonates. Pediatr Infect Dis J. 2012;31(12):1252-7. doi: 10.1097/INF.0b013e3182737427. [PubMed: 22982980].

5. Horn DL, Neofytos D, Anaissie EJ, Fishman JA, Steinbach WJ, Olyaei AJ, et al. Epidemiology and outcomes of candidemia in 2019 patients: data from the prospective antifungal therapy alliance registry. Clin Infect Dis. 2009;48(12):1695-703. doi: 10.1086/599039. [PubMed: 19441981].

6. Charsizadeh A, Mirhendi H, Nikmanesh B, Eshaghi H, Rahmani M, Farhang A, et al. Candidemia in children caused by uncommon species of Candida. Arch Pediatr Infect Dis. 2018;6(2). doi: 10.5812/pedinfect.11895.

7. Pfaller MA, Diekema DJ. Epidemiology of invasive candidiasis: A persistent public health problem. Clin Microbiol Rev. 2007;20(1):133-63. doi: 10.1128/CMR.00029-06. [PubMed: 17223626]. [PubMed Central: PMC1797637].

8. Pappas PG, Rex JH, Lee J, Hamill RJ, Larsen RA, Powderly W, et al. A prospective observational study of candidemia: epidemiology, therapy, and influences on mortality in hospitalized adult and pediatric patients. Clin Infect Dis. 2003;37(5):634-43. doi: 10.1086/376906. [PubMed: 12942393].

9. Velasco E, Bigni R. A prospective cohort study evaluating the prognostic impact of clinical characteristics and comorbid conditions of hospitalized adult and pediatric cancer patients with candidemia. Eur J Clin Microbiol Infect Dis. 2008;27(11):1071-8. doi: 10.1007/s10096-0080546-y. [PubMed: 18548295].

10. Blyth CC, Chen SC, Slavin MA, Serena C, Nguyen Q, Marriott D, et al. Not just little adults: candidemia epidemiology, molecular characterization, and antifungal susceptibility in neonatal and pediatric patients. Pediatrics. 2009;123(5):1360-8. doi: 10.1542/peds.2008-2055. [PubMed: 19403503].

11. Sadrossadati SZ, Ghahri M, Imani Fooladi AA, Sayyahfar S, Beyraghi S, Baseri Z. Phenotypic and genotypic characterization of Candida species isolated from candideamia in Iran. Curr Med Mycol. 2018;4(2):14-20. doi: 10.18502/cmm.4.2.64. [PubMed: 30324152] [PubMed Central: PMC6181062].

12. Barati M, Mirkalantari S, Ansari S, Salari S, Fattahi A. Determination of antimicotic susceptibility pattern of Candida species isolated from patients with symptomatic candiduria. J Res Med Sci. 2019;24:35. doi:10.4103/jrms.JRMS_880_18.[PubMed:31143236].[PubMed Central: PMC6521611]

13. Lotfali E, Kordbacheh P, Mirhendi H, Zaini F, Ghajari A, Mohammadi R, et al. Antifungal susceptibility analysis of clinical isolates of Candida parapsilosis in Iran. Iran J Public Health. 2016;45(3):322-8. [PubMed: 27141494]. [PubMed Central: PMC4851746].

14. Huang YC, Lin TY, Lien RI, Chou YH, Kuo CY, Yang PH, et al. Candidaemia in special care nurseries: Comparison of albicans and parapsilosis infection. J Infect. 2000;40(2):171-5. doi:10.1053/jinf.2000.0638. [PubMed: 10841095].

15. Juster-Reicher A, Flidel-Rimon O, Amitay M, Even-Tov S, Shinwell E, Leibovitz E. High-dose liposomal amphotericin B in the therapy of systemic candidiasis in neonates. Eur J Clin Microbiol Infect Dis. 2003;22(10):603-7. doi: 10.1007/s10096-003-0993-4. [PubMed: 13680398].

16. Barchiesi F, Caggiano G, Falconi Di Francesco L, Montagna MT, Barbuti $S$, Scalise G. Outbreak of fungemia due to Candida parapsilosis in a pediatric oncology unit. Diagn Microbiol Infect Dis. 2004;49(4):269-71. doi:10.1016/j.diagmicrobio.2004.03.011. [PubMed: 15313532]. 
17. Hegazi M, Abdelkader A, Zaki M, El-Deek B. Characteristics and risk factors of candidemia in pediatric intensive care unit of a tertiary care children's hospital in Egypt. J Infect Dev Ctries. 2014;8(5):624-34. doi: 10.3855/jidc.4186. [PubMed: 24820467].

18. Kara A, Devrim I, Mese T, Bayram N, Yilmazer M, Gulfidan G. The frequency of infective endocarditis in Candida bloodstream in fections: A retrospective study in a child hospital. Braz J Cardiovasc Surg. 2018;33(1):54-8. doi: 10.21470/1678-9741-2017-0049. [PubMed: 29617502]. [PubMed Central: PMC5873774].

19. Kafshdooz Jabari S, Chadeganipour M, Ghahri M, Mohammadi R. Etiologic agents of Candidemia in pediatric immunocompromised patients. Iran J Pediatr Hematol Oncol. 2016;6(4):209-15.

20. Colombo AL, Guimaraes T, Silva LR, de Almeida Monfardini LP, Cunha AK, Rady P, et al. Prospective observational study of candidemia in Sao
Paulo, Brazil: Incidence rate, epidemiology, and predictors of mortality. Infect Control Hosp Epidemiol. 2007;28(5):570-6. doi: 10.1086/513615. [PubMed: 17464917].

21. Steinbach WJ. Pediatric invasive candidiasis: Epidemiology and diagnosis in children. J Fungi (Basel). 2016;2(1). doi: 10.3390/jof2010005. [PubMed: 29376923]. [PubMed Central: PMC5753086].

22. Dutta A, Palazzi DL. Candida non-albicans versus Candida albicans fungemia in the non-neonatal pediatric population. Pediatr Infect Dis J. 2011;30(8):664-8. doi: 10.1097/INF.ob013e318213daof. [PubMed: 21372750].

23. Charsizadeh A, Mirhendi H, Nikmanesh B, Eshaghi H, Makimura K. Microbial epidemiology of candidaemia in neonatal and paediatric intensive care units at the Children's Medical Center, Tehran. Mycoses. 2018;61(1):22-9. doi: 10.1111/myc.12698. [PubMed: 28872714]. 